\title{
Editorial to RAIME 2008
}

\author{
Mohalingam Siva Kumar
}

Published online: 19 December 2008

(C) Springer-Verlag London Limited 2008

The current trend towards the globalization of industry challenges it to meet the changes in the development of customer tastes, by providing attractively priced and reliable new products. The rapid developments in mechanical engineering and research and the availability of the internet, causes fierce international competition, between manufacturers, which requires continuous research and the application of up-to-date technology, to sustain industries in this global market. The special edition of IJAMT, covers the recent advances in the application of mechanical and manufacturing engineering to this problem, and includes observational, experimental, and computational results.

The opening paper of the issue "Workability Studies on Al-5\% SiC Powder Metallurgy Composite During Cold Upsetting" is an experimental work in which the effect of particle size on the Al-SiC metal matrix composites has been deeply investigated under two different stress state conditions such as plane and triaxial. The paper "Artificial Neural Network a Tool for Predicting Failure Strength of Composite Tensile Coupons Using Acoustic Emission Technique" demonstrates the capability of backpropagation neural network to predict the ultimate strength of carbon/ epoxy tensile specimens. An attempt has been made in one of the papers to obtain optimum tolerance allocation of a complex assembly using Lagrange multipliers method with univariate search in which the components have alternative processes for manufacturing.

\footnotetext{
M. Siva Kumar $(\bowtie)$

Department of Mechanical Engineering,

National Engineering College,

K.R. Nagar,

Kovilpatti 628503 Tamil Nadu, India

e-mail: lawan_sisa@yahoo.com
}

One another paper highlights about the residual stresses in a typical boiler tube component and then it is characterized by nondestructive methods viz., magnetic Barkhausen noise (MBN) and X-ray diffraction (XRD) techniques which identify the type of damage such as corrosion and erosion. The paper entitled "Damage Characterization in SS 304 due to Monotonic Loading Using Infrared Thermography" discusses the method to use infrared thermographic technique for the characterization of damage accumulated in the material.

Experimental analysis on tensile properties of FRP with nano clay has been studied and reported in one of the papers. Interface bonding strength of particulate-reinforced metal matrix composites (Al-SiC) are investigated by joining process and its valuable results are reported in the paper "Evaluation of Interface Bonding Strength of Aluminium/Silicon Carbide." Experimental investigation of tensile creep behavior of polymer nanocomposites is reported in one among many.

Modeling and simulation of a parallel plate heat sink have been done in one of the papers using computational fluid dynamics. The use of the commercial FE software ABAQUS to determine the $J$-integral and stress intensity factor in austenitic stainless steel (AiSi 304) plates has been presented in one of the paper. The paper "CFD Simulation for Two Phase Mixing in 2D Fluidized Bed" discusses two phase (solid and gas) 2D fluidized bed reactor's flow pattern using FLUENT software and the simulation results are compared with the obtained experimental results. Finite element modeling of warping in smart piezoelectric noncircular shaft has been carried out using ANSYS software in one of the papers. One of the papers analyzes and compares the heat transfer properties of the nanofluids with the conventional fluids. 
I would like to take this opportunity to thank all the contributors of this special issue for their time, experience, and insights to these important topics. In particular, I would like to thank the Editor-in-chief Professor B. John Davies for giving me an opportunity to bring out such an issue. I am grateful for all the reviewers who carefully reviewed all the contributions in a relatively shorter time. Their valuable comments and suggestions certainly helped a lot to improve the quality of the issue. I trust that the readers surely enrich their knowledge by reading this special issue.

Professor M. Siva Kumar

Guest Editor 